\title{
Sequential search processes in long-term memory
}

\author{
CAROL L. KRUMHANSL, RICHARD C. MOHS, and RICHARD C. ATKINSON \\ Stanford University, Stanford, California 94905
}

\begin{abstract}
In an experiment employing the symbol-element recognition task (Mohs, Wescourt, \& Atkinson, 1975), subjects first learned six lists consisting of four words (elements) each. Each list was associated with a unique consonant (symbol). Subsequently, on each of a series of test trials, subjects were presented with one, two, or four symbol-element pairs. A positive response was required if all test words were correctly paired with their associated consonants and a negative response if any one test word was incorrectly paired with a consonant. Of primary concern was the way reaction time (RT) varied with number of pairs presented, the type of response required, and, on negative trials, the position of the mismatched pair in the test display. RT increased with the number of pairs presented on a trial and the increase was greater for positive than for negative trials. For negative pair trials, RT increased with the distance of the mismatched pair from the top of the test display. On negative trials in which the top pair in the test display was the mismatched pair, RT increased with the total number of pairs presented on the trial. A serial, probabilistic order of processing model is proposed to account for these results, and applications of the model to other paradigms are discussed.
\end{abstract}

Reaction time (RT) paradigms have frequently been used to investigate the memory processes underlying word recognition. In studies of recognition memory for words stored in long-term memory, subjects generally learn a single list of words and later are asked whether each of a series of test words was or was not a member of the memorized list. In order to make inferences about the memory processes underlying performance in this type of task, investigators have manipulated variables such as list structure and composition and examined the effect of these variables on RT and error rate. At present there is still disagreement as to whether strength models (e.g., Kintsch, 1970; Murdock, 1968; Postman, Jenkins, \& Postman, 1948; Wickelgren, 1975), memory search models (e.g., Anderson \& Bower, 1974; Shiffrin \& Atkinson, 1969; Tulving \& Thomson, 1971), or a model employing a combination of strength and search processes (Atkinson \& Juola, 1973, 1974) can best account for performance in this task.

Recently, a modified word recognition paradigm, called the symbolelement recognition paradigm, was employed by Mohs, Wescourt, and Atkinson (1975) to investigate the possible memory search processes involved in word recognition. In this paradigm subjects learn a number of distinct lists of words (called elements) and each list is associated with a unique consonant (called a symbol). On subsequent test trials subjects are given consonant-word (symbolelement) pairs

This research was supported by Grant MH-21747 from the National Institute of Mental Health and Grant NSF-EC 43997 from the National Science Foundation. Richard C. Mohs is now at the Department of Psychiatry and Behavioral Sciences, Stanford University Medical Center, Stanford, California. Requests for reprints should be sent to Carol L. Krumhansl, Department of Psychology, Stanford University, Stanford, California 94305. and are asked whether the test word was on the list labeled by the test consonant. Subjects are to respond "Yes" if the word is a member of the list associated with the consonant and "No" if the word is a member of one of the other memorized lists. Since all test words are taken from one of the memorized lists, the average frequency of presentation is the same for test words on both Yes and No trials. Thus, this paradigm would seem to minimize the possibility of responses based on differential memory strength values (which, presumably, are a function of frequency of word presentation) for positive and negative test items, and thereby maximize the probability of responses based on a search of a stored representation of the memorized list. The results of two experiments were reasonably well accounted for by a simultaneousentry search model. This model assumes that when a test display is presented, search processes are initiated simultaneously from two locations in memory. One search begins at a location where a representation of the test symbol is stored; this process determines whether the test element is associated with the test symbol. A second search process begins at a location in memory where a representation of the test element is stored; this process determines whether the test symbol is associated in memory with the test element. Anderson and Bower (1973, Chapter 12) have applied a similar model to RT data from a number of recognition memory experiments.

In the present experiment the symbol-element paradigm was employed to investigate the memory search processes involved in the verification of one or more associative relationships. Subjects memorized six word lists prior to a series of test trials. Each of the word lists consisted of four words (elements) and was associated with a unique consonant (symbol). On a test trial, subjects were presented with one, two, or four 
consonant-word pairs. For each pair, the subject was to determine if the word belonged with the consonant that it was paired with on that trial. If each word was correctly paired with its associated consonant (forming a "matched" pair), the subject was to respond "Yes." Otherwise, the subject was to respond "No." On No trials only one of the consonant-word pairs presenced was "mismatched," that is, only one pair consisted of a consonant paired with a word from a different list. In many respects, the single symbol-element task (Mohs et al., 1975) is similar to the sentence verification task used by Anderson and Bower (1973) and by Anderson (1974). During the study phase of the sentence verification task, subjects memorize a list of sentences constructed so that the subject or predicate of a given sentence may be repeated in other sentences. During a subsequent test phase, subjects are presented with a series of test sentences. All test sentences are formed using subjects and predicates that appear somewhere in the original memory lists, but not necessarily in the same sentence. Subjects are asked to say whether or not each test sentence appeared in the memorized list of sentences. During the test phase of such an experiment the subject is asked to judge the permissibility of certain subject-predicate relationships. Similarly, the test phase of the symbol-element experiment consists of verifying the permissibility of certain symbolelement relationships. The memory search models developed to account for performance in these two tasks are similar in many ways, although the interpretation of results from sentence verification experiments is heavily dependent upon assumptions made about the representation of sentences in memory. A sentence memory task analogous to the multiple symbol-element task studied here would require subjects to judge whether several sentences (that do not form a larger semantic unit such as a paragraph) were contained on a memorized list.

The primary purpose of the present experiment was to evaluate the interpair processing sequence when multiple items are presented for verification. Since an explicit model has been formulated for the processing of single symbolelement pairs (Mohs et al., 1975), the symbolelement pair was chosen as the unit for verification. A question of particular interest was whether subjects are able to verify list membership for more than one pair simultaneously or whether multiple pairs are processed in a strictly serial manner. The experiment investigated the interpair sequence by varying the number of pairs presented on a trial and by varying, on No trials, the position of the mismatched pair in the test display.

\section{METHOD}

\section{Subjects}

Sixteen female Stanford University students served as subjects. Each subject was paid $\$ 8$ for participating in four test sessions.

\section{Apparatus.}

The experiment was run using a programmable CRT display (Imlac Corporation PDS-1) interacting with a PDP-10 timesharing computer system. The test display appeared on the screen in green capital letters spaced at 2.8 characters $/ \mathrm{cm}$ and was viewed from approximately $50 \mathrm{~cm}$. Subjects made either a Yes or a No response on each trial by striking one of two specified keys on a typewriter-like keyboard located in front of the CRT screen.

\section{Materials}

Two complete sets of materials were used. Half of the subjects learned, and were tested on, each of these sets of materials. A set of materials consisted of six consonants and 24 words. The words, all six letters and two syllables in length, were selected so that acoustic, visual, and semantic confusions among the words were minimized. The words were drawn from the Thorndike-Lorge word list and have frequency counts of more than 20 per million. Consonants were selected so that visual confusions among them were minimized. The 24 words from each set of materials were divided into six lists of four words each. Each list of four words was uniquely associated with one of the six consonants from the set of materials.

On a test trial, the subject was presented with one, two, or four consonant-word pairs. In no case did a word or consonant appear twice on the same trial. The subject was instructed to respond "Yes" if all pairs presented were such that the test word was a member of the list designated by the test consonant Such a consonant-word pair is called "matched." The subject was to respond "No" if any one of the pairs presented was one in which the word was not a member of the list designated by the consonant. This type of consonant-word pair is called "mismatched." On trials with multiple consonant-word pairs, no more than one of the pairs presented was mismatched. Also, on trials in which a mismatched pair occurred, the word in the mismated pair was not taken from any of the lists designated by consonants presented on that trial. Trial types were defined in terms of the correct response (Yes or No), the total number of consonant-word pairs (one, two, or four), and, on No trials, the position of the mismatched pair in the test display.

For each set of materials, eight blocks of 24 trials were constructed. Within each block, Yes and No trials appeared equally of ten, and trials consisting of one, two, or four pairs appeared equally often. For No trials of a given length, mismatched pairs appeared equally often within the blocks. Each matched consonant-word pair appeared approximately twice in each block, and each possible mismatched pair appeared approximately once in all eight blocks. Each day a subject was given all eight trial blocks randomly ordered, with trials randomly ordered within the blocks.

\section{Procedure}

At least $18 \mathrm{~h}$ before the first test session, each subject was given a set of six index cards and a page of instructions. One list appeared on each of the cards. The words appeared in a column below the consonant used to designate the list. The subject was instructed to memorize the lists so that when given one of the six consonants she could respond with the words on the designated list in correct serial order. The subject was instructed to shuffle the cards while learning the lists so as not to learn the lists in any particular order.

Subjects were tested individually. At the start of each test session, the subject was tested for knowledge of the lists. All subjects were able to recall each of the lists in correct serial order with no errors.

After completing the recall test, the subject was seated in front of the CRT screen and given instructions about the task. The subject was told that there would be a series of test trials and the following sequence of events would occur on each trial: (a) The word "READY" would appear centered on the screen. (b) The subject would then press the spacebar on the keyboard 
to start the test sequence. (c) After a delay of $1 \mathrm{sec}$, the test display, consisting of one, two, or four pairs, would appear. (d) The test display would remain on the screen until the subject made a Yes or No response or until the time-out limit of $6 \mathrm{sec}$ was exceeded. (e) A feedback statement indicating correct response or incorrect response (or time-out error if the time limit was exceeded) would appear centered on the screen and remain there for $2.5 \mathrm{sec}$. The subject was instructed to respond "Yes" by pressing the " $M$ " key if all consonant-word pairs on the trial were matched and respond "No" by pressing the " $C$ " key if any one of the consonant-word pairs was mismatched. Subjects were informed that, at most, one of the consonant-word pairs would be mismatched on multiple consonant-word pair trials. For all pairs the test consonant was displayed two spaces to the left of the test word. On multiple pair trials, the pairs were listed vertically, with a vertical distance of $.38 \mathrm{~cm}$ between the test pairs. For each number of consonant-word pairs, the configuration was adjusted vertically so that the display was centered on the screen. The instructions emphasized that subjects were to respond as quickly as possible while trying to avoid errors.

At all test sessions 10 practice trials were given, followed immediately by the test trials. Subjects received approximately $5 \mathrm{trials} / \mathrm{min}$. The four test sessions were scheduled on consecutive days for each subject.

\section{RESULTS}

A summary of the data is presented in Figures 1 and 2. Although there were large practice effects from Day 1 to Days 3 and 4 , these effects were only minimal after Day 3. Accordingly, the data from Days 3 and 4 were combined and the data from Day 1 and from Days 3 and 4 combined are shown. Reaction times from trials on which an incorrect response was made are not included. The data from two subjects were eliminated due to abnormally large error rates (over $25 \%$ ), and the mean RTs in Figures 1 and 2 are the mean of the subject mean RTs for the remaining 14 subjects.

In Figure 1, mean RTs for Day 1 and Days 3 and 4 combined are plotted against the number of pairs presented on the trial. The solid line represents the RTs for trials on which Yes was the correct response; the dashed line represents the RTs for trials on which No was the correct response. Error rates are affixed to the RT points. In Figure 2, the results for No trials are plotted as a function of the position of the mismatched pair in the test display. On multiple pair trials, the consonant-word pairs were listed vertically in the test display, and position in the test display refers to the vertical distance from the top of the test display. The point labeled $1 \mathrm{~N}$ represents the mean RT for trials on which No was the correct response and the trial consisted of one consonant-word pair. The line labeled $2 \mathrm{~N}$ represents the RTs for trials on which No was the correct response and the trial consisted of two pairs. Similarly, the line labeled $4 \mathrm{~N}$ represents No trials consisting of four pairs. As in Figure 1, error rates are affixed to the RT points.

Mean RTs for trials on which an incorrect response was made are shown in Figure 3 for Day 1 and for Days 3 and 4 combined. Mean RTs are plotted against the total number of pairs presented on the trial. The solid line corresponds to Yes trials on which a No response was given; the dashed line corresponds to No trials on wilich a Yes response was given. The number of observations for each point are affixed to the RT points. Note that error rates were greater for No trials, and incorrect RTs on these trials were comparable to correct RTs on Yes trials.

For RTs from trials on which a correct response was made, an analysis of variance was computed using three within-subjects factors: (a) Test Days (Day 1, 2, 3, or 4) (b) Response (Yes vs. No), and (c) Number of Pairs presented on the trial (one, two, or four). Subjects

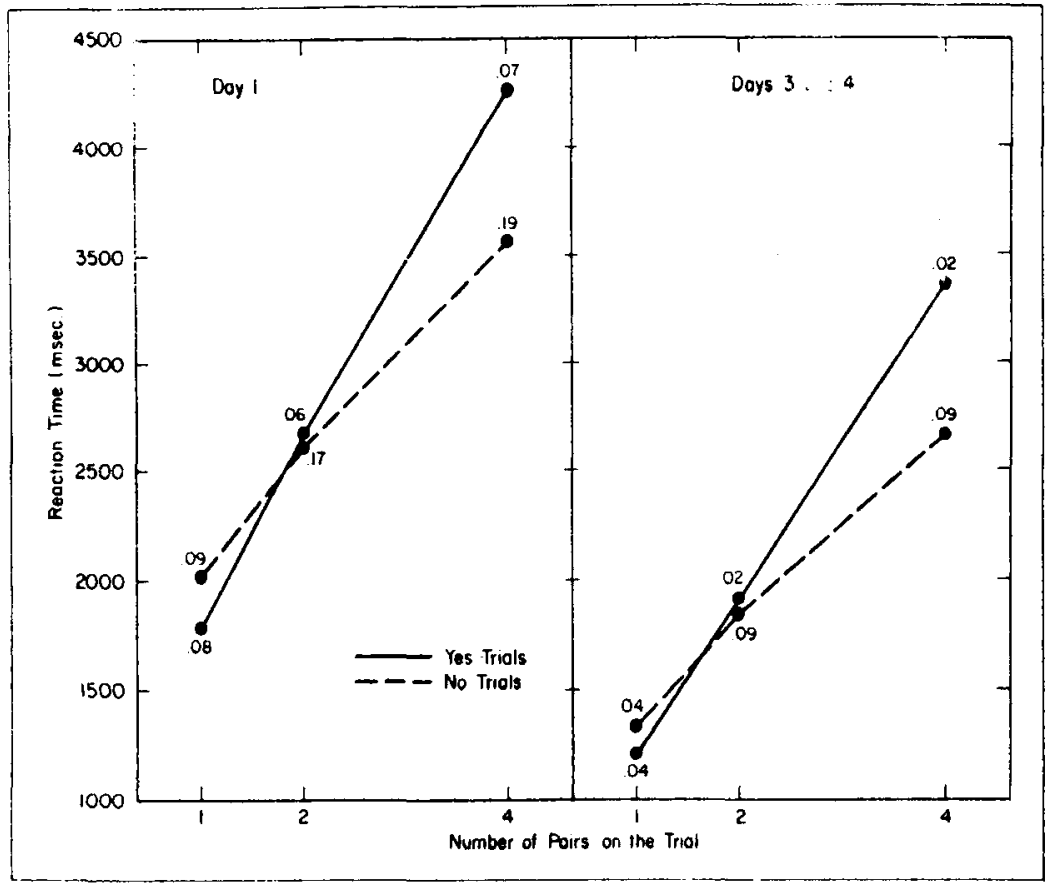

Figure 1. Mean RTs for Day 1 and Days 3 and 4 combined as a function of the number of pairs presented. Error rates are affixed to $R T$ points. 


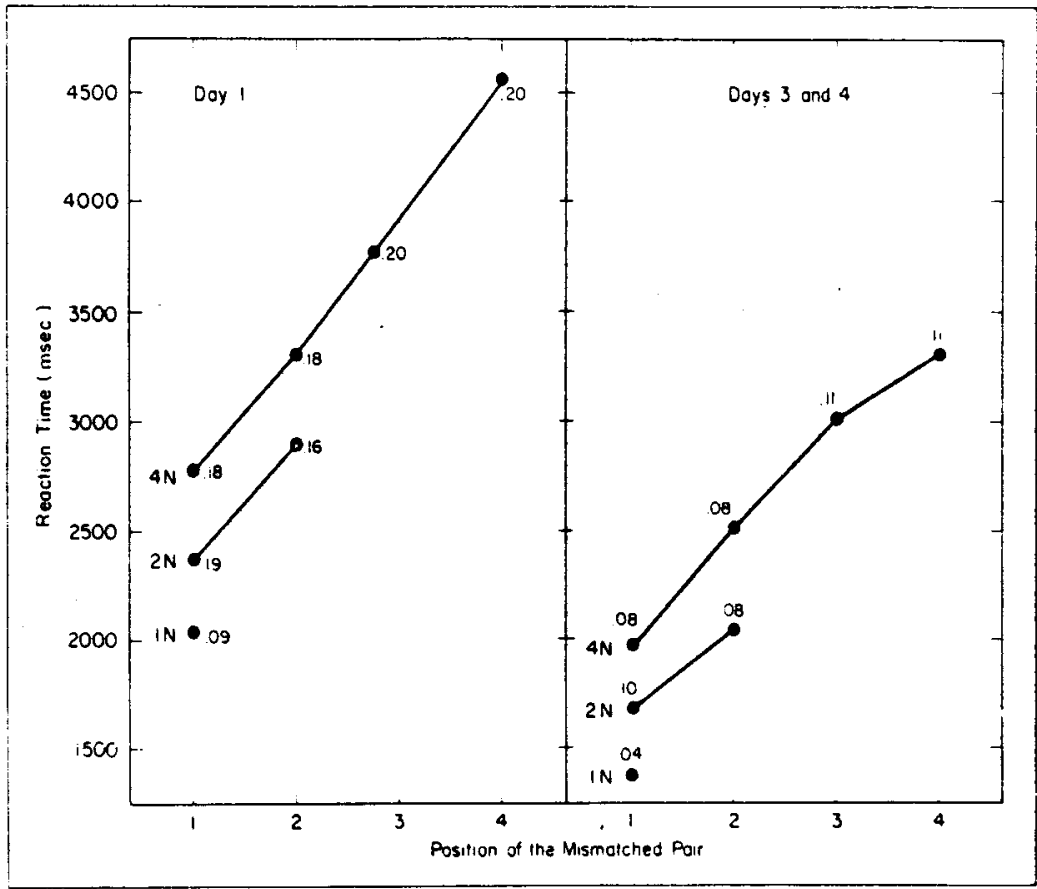

Figure 2. Mean RTs for Day 1 and Days 3 and 4 combined as a function of the position of the mismatched pair. Error rates are affixed to $R T$ points.

were divided in the analysis according to the set of materials they had learned, and subject means were used as scores. The effect of Set of Materials was not significant in its main effect or any of its interactions, and subsequent analyses did not include this betweensubjects factor. The analysis indicated a significant practice effect over Days $[F(3,36)=49.53, p<.001]$ and a significant Response (Yes vs. No) effect $[F(1,12)=$ $16.25, \mathrm{p}<.01]$. The Number of Pairs on the trial also yielded a significant effect $[F(2,24)=498.38$, $\mathrm{p}<.001$ ] , of which $99.9 \%$ was accounted for by a linear contrast. Finally, two interaction effects were significant; Days by Number of Pairs $[F(6,72)=6.66$, $p<.001]$ and Response by Number of Pairs $[F(2,24)=$ $177.99, p<.001]$. The first of these interactions is seen in Figure 1 as the decreased slopes for both Yes and No responses for the later test days compared with the first test day. The second of these interactions is seen as the greater slopes for Yes responses than for No responses. All other interaction effects were not significant.

As Figure 2 shows, the pattern of results on No trials was similar for Day 1 and for Days 3 and 4 combined. For simplicity we will present statistical tests only for the data from Days 3 and 4, where the effects are smaller; these analyses will serve to demonstrate the

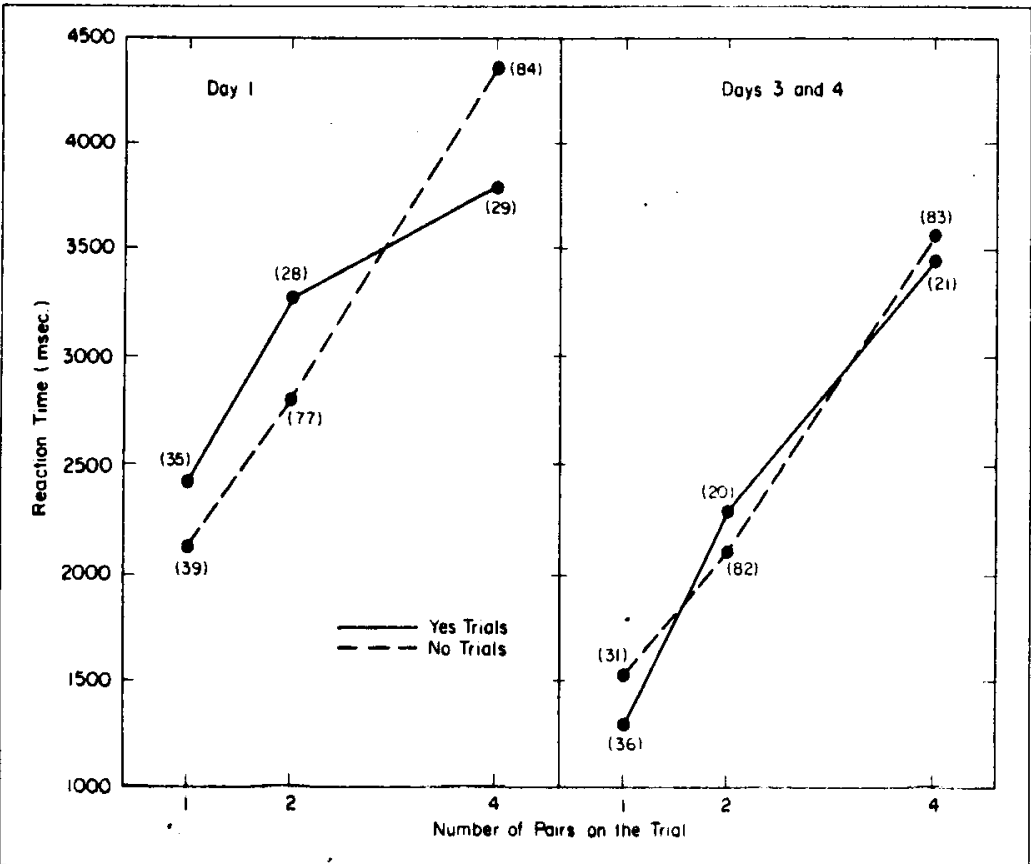

Figure 3. Mean RTs for Day 1 and Days 3 and 4 combined for trials on which an incorrect response was made. Number of observations are affixed to RT points. 
robustness of the differences shown in Figure 2. A one-way analysis was computed using subject means for trials consisting of four consonant-word pairs, but varying in the position of the mismatched pair in the display. The Position of the Mismatched Pair was found to have a significant effect $[F(3,39)=28.49, p<.001]$, of which $98 \%$ was accounted for by a linear contrast. Deviations from the linear trend were not significant. This effect is seen in the right-hand panel of Figure 2 as the linear increase in RT as the mismatched pair was moved from the top of the test display (Position 1) to the bottom (Position 4) on $4 \mathrm{~N}$ trials. A similar increase is seen on $2 \mathrm{~N}$ trials. A second one-way analysis of variance was computed on the mean RTs from trials on which the pair in the first position was mismatched; the Number of Pairs was a within-subjects factor with three levels (one, two, or four). The Number of Pairs was found to have a significant effect $[F(2,26)=11.62, p<.001]$, even though the first pair was the mismatched pair for all cases included in the analysis. This effect is seen in Figure 2 as the difference in RTs for $1 \mathrm{~N}, 2 \mathrm{~N}$, and $4 \mathrm{~N}$ trials when the position of the mismatched pair is fixed at the first position.

Since subjects in this experiment learned the words on each list in a fixed order, we can look at RT as a function of the serial position (list position) from which test words were drawn. On trials consisting of one pair, the test word had Serial Position 1, 2, 3, or 4 on its memorized list. On multiple pair trials, words were drawn from several combinations of serial position; this prevented subjects from developing special strategies for checking only certain list positions. The serial position data, however, are based on only those trials in which all words were taken from the same serial position on their respective memorized lists. On trials consisting of two pairs, the two words were both drawn from Serial Position 1, 2, 3, or 4 on their respective memorized lists. Similarly, on trials consisting of four pairs, the words all had Serial Position 1,2,3, or 4. One-half of the $2 \mathrm{~N}$ and $4 \mathrm{~N}$ trials were of this type. The serial position data for all 4 days combined is shown in Figure 4. 1Y, 2Y, and $4 \mathrm{Y}$ refer to Yes trials of Lengths 1,2 , and 4 , respectively; $1 \mathrm{~N}, 2 \mathrm{~N}$, and $4 \mathrm{~N}$ refer to No trials of Lengths 1,2 , and 4 , respectively. Error rates are affixed to the RT points.

An analysis of variance was computed using the subject mean RTs for all 4 days combined. The Serial Position effect was found to be significant $[F(3,39)=9.06$, $\mathrm{p}<.001$ ], $94 \%$ of which was accounted for by a linear contrast. As before, Response (Yes vs. No), Number of Pairs (one, two, or four), and Response by Number of Pairs were significant. No other interactions in the analysis proved to be significant.

\section{DISCUSSION}

Performance on the multiple symbolelement recognition task can be summarized as follows: (a) RT increased linearly with the total number of pairs presented on a trial. (b) The increase in RT with number of pairs was greater for Yes trials than for No trials; the ratio of Yes to No slopes was about 1.65. (c) On multiple pair No trials, RT was a linear function of the position of the mismatched pair in the test display; the slope of this function was less than the slope of the function relating Yes RTs to the total number of pairs in the trial. (d) For No trials in which the first pair was mismatched, RT increased with the total number of pairs in the trial. (e) Error rates were greater for No trials, and incorrect RTs on these trials were comparable

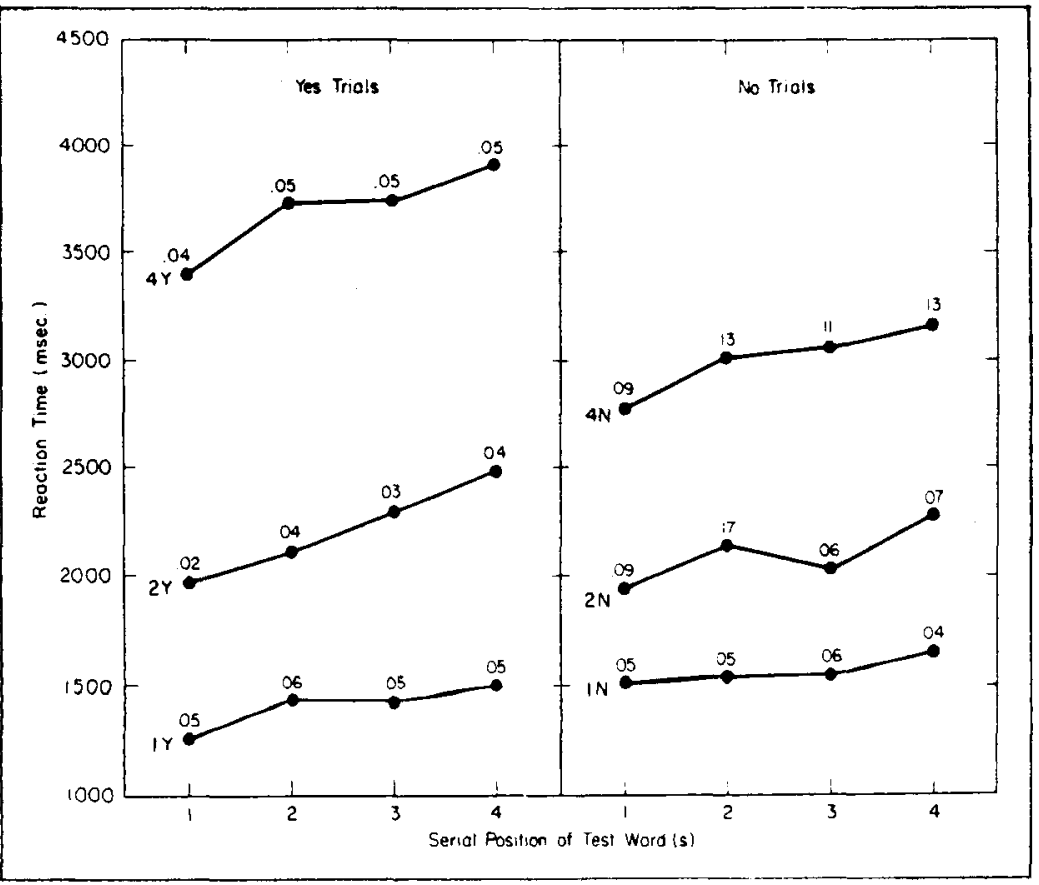

Figure 4 Mean RTs for all days combined as a function of the serial position of the test word(s). Error rates are affixed to $\mathrm{RT}$ points. 
to correct RTs on Yes trials. (f) RT increased with the serial position of the test words in their memorized lists.

\section{A Sequential Model ·}

These results suggest certain characteristics of a model for the processing of multiple test items. For the sake of clarity, we will present the model in the context of the results from the present experiment. However, the model is potentially applicable to the processing of multiple test items in other paradigms such as word recognition (Atkinson \& Juola, 1973) and sentence verification (Anderson, 1974). Later we will discuss some issues in applying the model to other paradigms.

The difference between Yes and No slopes suggests that subjects generally terminated processing as soon as a mismatched pair was found (Sternberg, 1969). The increase in RT on No multiple pair trials as the mismatched pair moved from the top to the bottom of the test display indicates that processing generally began at the top of the display and proceeded downward. Two results, however, argue against processing in a strictly top-to-bottom order. First, for multiple pair trials, the slope of the function relating No RT to the position of the mismateched pair was less than the slope of the function relating Yes RT to the total number of pairs presented. Second, on No trials in which the first pair was the mismatched pair, RT increased with the total number of pairs presented. If processing had been in a strictly top-to-bottom order, the functions relating No RT to position of the mismatched pair for the different total number of pairs presented would be overlapping lines with slopes equal to the slope of the function relating Yes RT to the total number of pairs presented. A similar argument against a strict processing order is made by Stemberg (1967).

Various models were investigated for the interpair processing sequence. The most successful model was one that assumes serial processing on pairs, with trial-to-trial variations in the order of pair processing for multiple pair trials. The model also assumes that processing terminates as soon as a mismatched pair is found. Here we should note that Townsend $(1971,1974)$ has shown that it is difficult to distinguish uniquely between parallel and serial processes on the basis of RT data; therefore, it is possible that a parallel processing model formally equivalent to the serial processing model proposed here could be formulated. The present model is appealing for several reasons. Most important is that it accounts for many aspects of the data obtained in the present experiment. In addition, the model is relatively simple conceptually and is in good.agreement with subjects' introspections about their performance on this task.

A reasonably good fit to the data was achieved using one order of processing parameter $\pi$. On trials consisting of two pairs, $\pi$ is the probability that the first pair is processed first, $(1-\pi)$ the probability that the second pair is processed first. On trials consisting of four pairs, $\pi$ is the probability that the first two pairs are processed first, $(1-\pi)$ the probability that the second two pairs are processed first. Within the two pairs, $\pi$ is the probability that the first pair is processed first, $(1-\pi)$ the probability that the second pair is processed first. If $\pi<1$, the model is consistent with the result that RT on No trials in which the first pair was mismatched increased with the total number of pairs in the trial. If, in addition, $\pi>.5$, the model predicts a positive slope for RT plotted against the position of the mismatched pair on No trials, the slope being less than the slope for RT plotted against the total number of pairs on Yes trials. Again, this is consistent with the data.

The other parameters of the model are $\gamma, \bar{\gamma}, \rho, \alpha$, and $\beta . \gamma$ is the sum of the times to encode a test pair and determine that the pair is matched. $\bar{\gamma}$ is the sum of the times to encode a test pair and determine that the pair is mismatched. $\rho$ is the sum of the times to organize and execute a response. $\alpha$ is the probability of making an error in the processing of a matched pair (deciding the pair is mismatched). $\beta$ is the probability of making an error in the processing of a mismatched pair (deciding the pair is matched). A more detailed description of the model is given in the Appendix.

Ten mean RTs and 10 error rates from Days 3 and 4 combined were used to estimate parameters. The estimated values of the parameters and the-predicted and observed values of correct RTs, incorrect RTs, and error rates for all trial types are given in Table 1. The notation used in Table 1 for trial type is the same as that used in Figure 4. The number following the trial type on No trials with more than one consonant-word pair indicates the position of the mismatched pair in the test display. The model is consistent with the linear increase found in RT as the number of pairs increased, the increase being greater for Yes than No trials. The model is also consistent with the pattern of results on multiple pair No trials as the position of the mismatched pair in the test display varied. The model predicts that incorrect RTs should be comparable to RTs for correct trials of the same response and should show no effect of the position of the mismatched pair in the test display on No trials. Predicted incorrect RTs and error rates are generally in accord with the experimental results. The fit of the model to the experimental results of Days 3 and 4 combined is shown in Figure 5. The lefthand panel shows predicted and observed RTs for Yes and No trials as a function of the total number of pairs presented on the trial; the right-hand panel shows predicted and observed RTs for No trials as a function of the position of the mismatched pair in the test display. The model with adjusted parameter estimates also achieves a good fit to the experimental results of Day 1 .

The model just presented characterizes the interpair processing sequence. The experiments reported by Mohs et al. (1975) were concerned with the processing done on a single symbol-element pair. RT data were used to 
Table 1

Predictions of the Model and Experimental Results

Shown are Predicted and Observed Correct RTs, Incorrect RTs, and Error Rates for Days 3 and 4

\begin{tabular}{|c|c|c|c|c|c|c|c|c|}
\hline \multirow[b]{2}{*}{ Trial Type } & \multicolumn{3}{|c|}{ Correct RTs } & \multicolumn{3}{|c|}{ Incorrect RTs } & \multicolumn{2}{|c|}{ Error Rates } \\
\hline & Predicted & Observed & $\begin{array}{l}\text { Number } \\
\text { Observed }\end{array}$ & Predicted & Otserved & $\begin{array}{l}\text { Number } \\
\text { Observed }\end{array}$ & Predicted & Observed \\
\hline $1 Y$ & 1147 & 1214 & 860 & 1455 & 1278 & 36 & .010 & .040 \\
\hline $2 Y$ & 1916 & 1943 & 876 & 1840 & 2237 & 20 & .020 & .022 \\
\hline $4 Y$ & 3454 & 3361 & 875 & 2609 & 3393 & 21 & .039 & .023 \\
\hline $1 \mathrm{~N}$ & 1455 & 1353 & 865 & 1147 & 1527 & 31 & .080 & .035 \\
\hline $2 \mathrm{Nl}$ & 1601 & 1674 & 403 & 1916 & 2200 & 45 & .079 & .100 \\
\hline $2 \mathrm{~N} 2$ & 2078 & 2058 & 411 & 1916 & 1967 & 37 & .079 & .083 \\
\hline $4 \mathrm{~N} 1$ & 1893 & 1975 & 206 & 3454 & 3671 & 18 & .078 & .080 \\
\hline $4 \mathrm{~N} 2$ & 2370 & 2503 & 207 & 3454 & 3614 & 17 & .078 & .076 \\
\hline $4 \mathrm{~N} 3$ & 2847 & 3009 & 200 & 3454 & 3627 & 24 & .078 & .107 \\
\hline $4 \mathrm{~N} 4$ & 3324 & 3280 & 200 & 3454 & 3588 & 24 & .078 & .107 \\
\hline
\end{tabular}

Note-The predicted values are based on the following parameter estimates: $\gamma=769, \bar{\gamma}=1077, \rho=378, \pi=.81, \alpha=.01, \beta=.08$.

investigate the structure of the memory representation for word lists and the processes by which list member. ship was evaluated. The results of those experiments indicated that word lists were represented in memory as a set of words associated serially with the list symbol linked to the first word in the list. The processing model most consistent with their results was a simultaneousentry model. This model assumes that both the test symbol and the test word serve as entry points into memory. Search processes are initiated simultaneously at both entry points and the search that finishes first determines the RT for that trial. The processing model, taken together with the proposed serially ordered memory representation, predicts serial position effects for both Yes and No trials. That 's, RT should increase with the distance of the test element from the top of its list. The prediction was consistent with the results of the single symbol-element experiment. In the multiple symbol-element experiment, RT was also found to increase with the serial position of the word in its memorized list. The simultaneous-entry model in conjunction with the serially ordered memory representation is applicable to the processing of single symbolelement pairs contained within a multiple symbolelement display.

\section{Generality of the Model}

The sequential model is potentially applicable to a variety of tasks in which the subject is presented multiple test items. The model, when applied to other tasks, can serve as a framework in which to describe certain characteristics of the multiple item processing sequence. In applying the model to other tasks, several issues must be considered. The most basic concern is whether the processing of multiple items in a particular paradigm can be characterized as a serial process. What evidence there is suggests that even very simple tasks, such as making a lexical decision, require serial processing of individual test items when multiple test items are presented (Schvaneveldt \& Meyer, 1973). A second issue is how the sequential processing order might be affected by certain variables relevant to a particular paradigm. For example, it is likely that the processing order for verifying multiple sentences would depend on the semantic relationships between the items on multiple sentence trials; that is, if sentences are arranged in logical order to form paragraphs, subjects might be induced to adopt a strictly top-to-bottom processing order.

Possibly the most interesting aspect of any application of the model is a characterization of the relationship between sequential processing times. For the

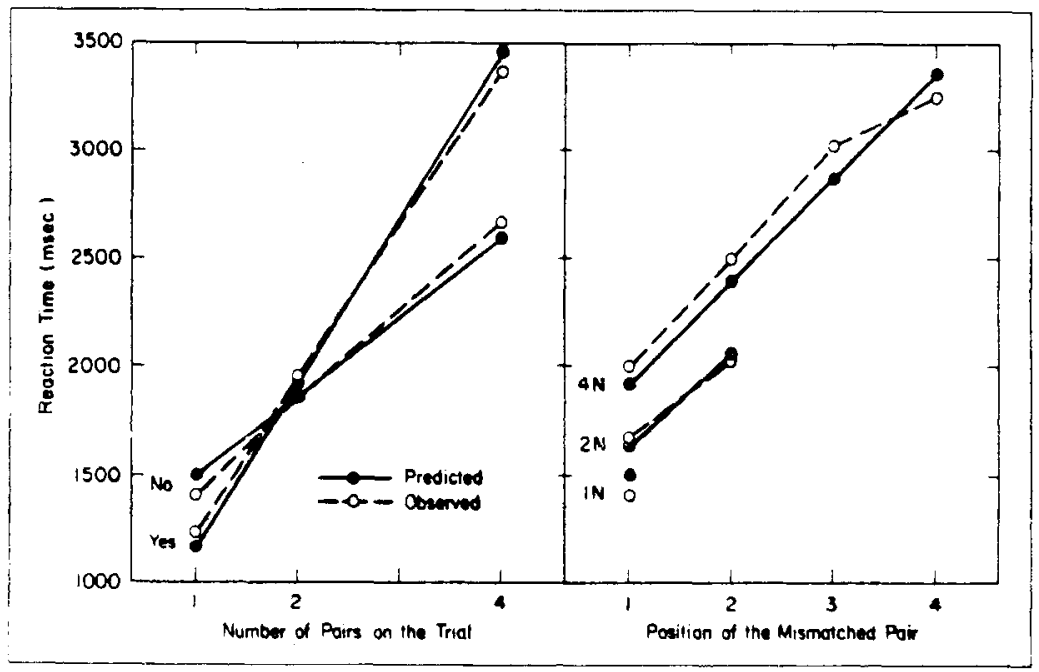

Figure 5. Predicted and observed mean RTs for Days 3 and 4 combined as a function of the number of pairs presented and as a function of the position of the mismatched pair. 
present experiment, two processing time parameters were sufficient. One parameter represented the time to process a matched pair, the other the time to process a mismatched pair. These times were assumed to be independent of the number or nature of previous items processed. In other tasks, however, the model might require modification to reflect the sequential dependencies between processing operations. Schvaneveldt and Meyer (1973), for example, showed that the time to decide that a letter string is a word decreased if a highly associated word was processed just previously. Similar processing dependencies might be found in a sentence verification task if a subject or predicate was repeated in adjacent sentences. The sequential model provides a convenient way of describing such dependencies.

\section{APPENDIX}

The parameters of the probabilistic order of processing model are described in the text. In addition, let $n=$ the total number of pairs on the trial and let $m=$ the position of the mismatched pair in the test display on No trials.

The model predicts that a "Yes" response is executed only after all of the presented pairs have been processed. The time to make a correct "Yes" response on a trial with $\mathrm{n}$ matched pairs is:

$$
\text { RT("Yes";Yes,n) }=\mathrm{n} \gamma+\rho \text {. }
$$

The probability of making a correct "Yes" response on a trial with $\mathbf{n}$ pairs is:

$$
\text { P("Yes"; Yes,n) }=(1-\alpha)^{n} \text {. }
$$

The time to make an incorrect "Yes" response (responding "Yes" when the correct response is "No") on a trial with $n$ pairs is independent of the position of the mismatched pair and is given by:

$$
\mathrm{RT}(\text { ("Yes";No,n) }=\mathrm{n} \boldsymbol{\gamma}+\rho \text {. }
$$

The probability of making an incorrect "Yes" response on a trial with $n$ pairs is:

$$
\mathrm{P}(\text { “Yes";No,n })=\beta(1-\alpha)^{\mathrm{n}-1} \text {. }
$$

The model predicts that a "No" response is executed as soon as a pair is judged to be mismatched; furthermore, the response mechanism can be triggered either by a correctly judged mismatched pair or an incorrectly judged matched pair. Exact expressions for RTs to make a correct "No" response are quite complicated due to the number of ways a "No" response can be initiated. However, reasonably simple approximate expressions can be written if it is assumed that $\alpha \approx 0$. The approximate time to make a correct "No" response on trials with n pairs in which the mismatched pair is in position $\mathrm{m}$, assuming $\alpha \approx 0$, is given by:

$$
\mathrm{RT}(\text { "No";No,n,m) } \approx \bar{\gamma}+[\mathrm{n}-\mathrm{m}+(2 \mathrm{~m}-\mathrm{n}-1) \pi] \gamma+\rho-
$$

The approximate time to make an incorrect "No" response (responding "No" when the correct response is "Yes") on a trial consisting of $n$ pairs, assuming $\alpha \approx 0$, is given by:

$$
\mathrm{RT} \text { ("No"; Yes,n) } \approx \bar{\gamma}+(\mathrm{n}-1) \gamma / 2+\rho \text { - }
$$

\section{REFERENCES}

ANDERson, J. R. Retrieval of propositional information from long-term memory. Cognitive Psychology, 1974, 4, 451-474.

ANDERSON, J. R., \& Bower, G. H. Human associative memory. Washington: Winston, 1973.

ANDERSon, J. R., \& Bower, G. H. A propositional theory of recognition memory. Memory \& Cognition, 1974, 2, 406-413.

Atkinson, R. C., \& Juola, J. F. Factors influencing speed and accuracy of word recognition. In S. Kornblum (Ed.), Attention and performance IV. New York: Academic Press, 1973.

Atkinson, R. C., \& Juola, J. F. Search and decision processes in recognition memory. In D. H. Krantz, R. C. Atkinson, \& P. Suppes (Eds.), Contemporary developments in mathematical psychology. San Francisco: Freeman, 1974.

KintsCh, W. Models for free recall and recognition. In D. A. Norman (Ed.), Models of human memory. New York: Academic Press, 1970.

Mohs, R. C., Wescourt, K. T., \& Atrinson, R. C. Search processes for associative structures in long-term memory. Journal of Experimental Psychology: General, 1975, 104, 103-121.

Murdock, B. B., JR. Modality effects in short-term memory: Storage or retrieval? Joumal of Experimental Psychology, $1968,77,79-86$.

Postman, L.. Jenkins, W. O., \& Postman, D. L. An experimental comparison of active recall and recognition. American Journal of Psychology. 1948, 61, 511-519.

Schvaneveldt, R. W., Meyer, D. E. Retrieval and comparison processes in semantic memory. In S. Kornblum (Ed.). Attention and performance IV. New York: Academic Press. 1973.

Shiffrin, R. M., \& Atkinson, R. C. Storage and retrieval processes in long-term memory. Psychological Review, 1969, 76, 179-193.

Sternberg, S. Retrieval of contextual information from memory. Psychonomic Science, 1967, 8, 55-56.

Sternberg, S. Memory scanning: Mental processes revealed by reaction-time experiments. American Scientist, 1969. 57, 421-457.

Townsend, J. T. A note on the identifiability of parallel and serial processes. Perception \& Psychophysics, 1971, 10, $161-163$.

TownsEnd, J. T. Issues and models concerning the processing of a finite number of inputs. In B. H. Kantowitz (Ed.). Human information processing: Tutorials in performance and cognition. Hillsdale, N.J: Erlbaum, 1974.

Tulving, E., \& Tromson, D. M. Retrieval processes in recognition memory: Effects of associative context. Journal of Experimental Psychology, 1971, 87, 116-124.

WiCKELGREN, W. A. Dynamics of retrieval. In D. Deutsch and J. A. Deutsch (Eds.), Short-term memory. New York: Academic Press, 1975.

(Received for publication October 8, 1975; revision accepted December 15, 1975.) 